\title{
Long-term Outcome of Triple Stapling Resection and J Pouch Anal Stapling Anastomosis for Ulcerative Colitis
}

\author{
HIDEJIRO KAWAHARA ${ }^{1,2}$ and NOBUO OMURA ${ }^{2}$ \\ ${ }^{1}$ Department of Surgery, Kashiwa Hospital, Jikei University School of Medicine, Chiba, Japan; \\ ${ }^{2}$ Department of Surgery, Nishisaitama-chuo National Hospital, Saitama, Japan
}

\begin{abstract}
Background: We previously reported laparoscopic total proctocolectomy with J pouch anal anastomosis, which was created at the dentate line by our original procedure using staplers, Triple Stapling Resection and J pouch Anal Stapling Anastomosis (TSRJASA), for ulcerative colitis (UC) patients. UC patients have undergone TSRJASA since it was introduced in our institution. However, the long-term outcome of TSRJASA for UC patients has not been elucidated. Patients and Methods: From January 2014 to December 2018, fourteen patients with ulcerative colitis, including three cases of concomitant cancer, who underwent TSRJASA were enrolled in this study. Anal manometry was performed using the Pock Monitor GMMS-100 system (STAR MEDICAL, INC., Tokyo, Japan) one and two years after surgery. Maximum resting pressure, maximum squeeze pressure, and the length of the high-pressure zone were measured. Fecal incontinence was evaluated using the Wexner incontinence questionnaire. Results: J pouch anal anastomosis was created at the dentate line in all patients. In a manometric examination two years after surgery, maximum resting pressure was 75.3 (54-88) $\mathrm{mm} \mathrm{Hg}$, maximum squeeze pressure was 125.0 (90-160) $\mathrm{mm} \mathrm{Hg}$, and the length of the high-pressure zone was 39.6 (35-42) mm. Wexner score was 2.8 (1-4). Conclusion: TSRJASA is a useful procedure for UC patients given its acceptable defecation function.
\end{abstract}

When rectal transection is attempted immediately above the anal canal with a $60-\mathrm{mm}$ length linear stapler, the anterior wall of the rectum just above the anal canal cannot be transected

This article is freely accessible online.

Correspondence to: Hidejiro Kawahara, MD, Ph.D., Department of Surgery, Kashiwa Hospital, Jikei University School of Medicine, 163-1 Kashiwashita, Kashiwashi, Chiba 277-8567, Japan. Tel: +81 471641111 Ext. 3421, Fax: +81 471633488 e-mail: kawahide@outlook.jp

Key Words: Stapling resection, stapling anastomosis, iliac pouch, ulcerative colitis, rectourethral muscle. anatomically (1). However, after cutting the rectourethral muscle using a 30-mm length stapler, rectal transection can easily be performed immediately above the anal canal using a $30-\mathrm{mm}$ length stapler twice. After rectal transection immediately above the anal canal, J pouch anal stapling anastomosis can be created at the dentate line because the oral side of the anal canal from the dentate line has been resected using a 25-mm circular stapler (2). We previously reported this procedure named Triple Stapling Resection and J pouch Anal Stapling Anastomosis (TSRJASA) in 2017 (2). However, long-term outcome of TSRJASA for patients with UC has not been elucidated. Therefore, to elucidate the long-term outcome of TSRJASA for patients with UC, we performed the present retrospective study in our institution.

\section{Patients and Methods}

Patients. The Ethics Committee for Biomedical Research of the Jikei Institutional Review Board approved the protocol [30-164(9185)]. From January 2014 to December 2018, fourteen patients with ulcerative colitis, including three cases with concomitant cancer, who underwent TSRJASA were retrospectively enrolled in this study. Anal manometry was performed using the Pock Monitor GMMS-100 system (STAR MEDICAL, INC., Tokyo, Japan) one year and two years after surgery. Maximum resting pressure, maximum squeeze pressure, and the length of the high-pressure zone were measured. Fecal incontinence was evaluated using the Wexner incontinence questionnaire.

Surgical technique. After laparoscopic total mesorectal mobilization to the anal canal, rectal transection was performed at the upper edge of the anal canal by firing three sets of $30-\mathrm{mm}$ long staplers (Figure 1). If a patient is male, the purpose of the first firing is to incise the rectourethral muscle to separate the rectum and urethra (Figure 2). After the rectal transection, $\mathrm{J}$ pouch anal anastomosis was performed by the double stapling technique using a $25-\mathrm{mm}$ circular stapler through the anus (Figure 3). J pouch anal anastomosis is created at dentate line because the oral side of the anal canal from the dentate line is resected by a circular stapler (Figure 4). A temporary stoma was created in all patients and was closed 3 to 6 months postoperatively (3).

Statistical analysis. Continuous variables are expressed as the mean and range. The paired $t$-test was used for the comparison of continuous variables. A $p$-Value of less than 0.05 indicated statistical significance. All data were analyzed with IBM SPSS Statistics, version 24.0 (IBM Japan, Ltd, Tokyo, Japan). 


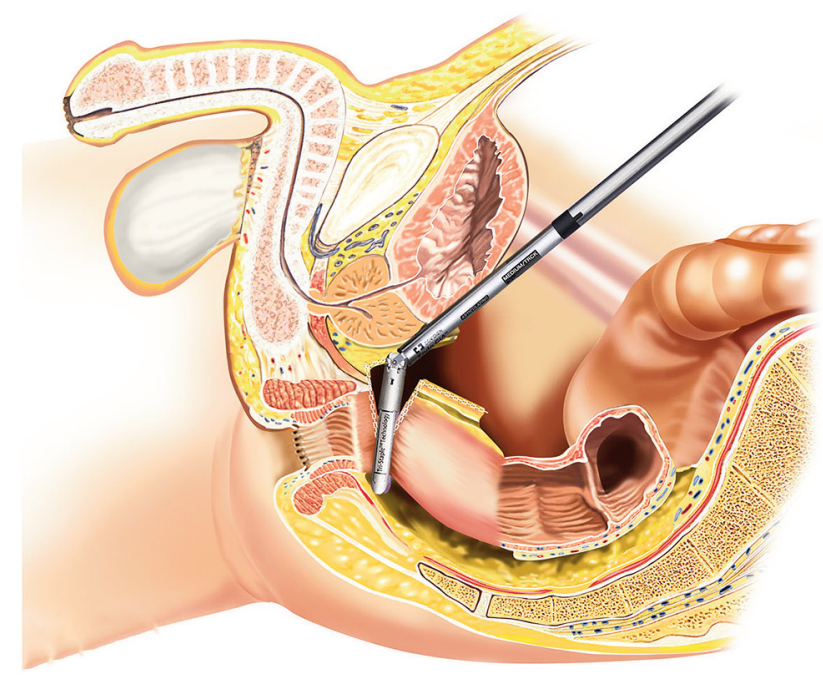

Figure 1. Schema of rectal transection using the stapler. Rectal transection is performed at the upper edge of the anal canal by firing three sets of $30 \mathrm{~mm}$-long staplers.

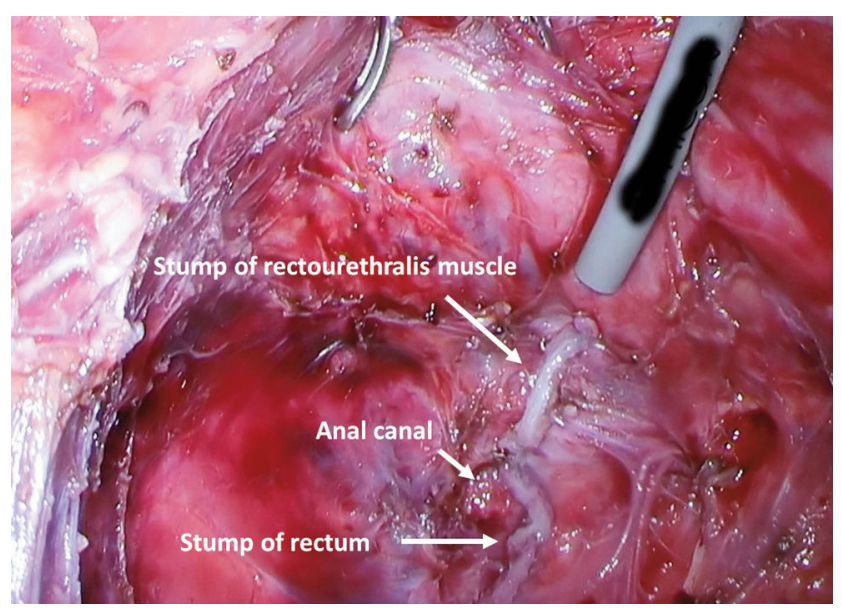

Figure 2. View of rectal transection. The stump of rectourethral muscle is shown closely cranial side of the rectal stump in the anal canal.

\section{Results}

The mean age of fourteen patients, including ten males and four females, was 40.7 (19-77) years (Table I). J pouch anal anastomosis was created at the dentate line in all patients. In a manometric examination after surgery, maximum resting pressure was 71.7 (52-85) $\mathrm{mmHg}$ one year after surgery and 75.3 (54-88) $\mathrm{mm} \mathrm{Hg}$ two years after surgery. Maximum squeeze pressure was 112.4 (87-141) $\mathrm{mm} \mathrm{Hg}$ one year after surgery and 125.0 (90-160) $\mathrm{mmHg}$ two years after surgery. The length of the high-pressure zone was 33.4 (30-35) mm one year after surgery and 39.6 (35-42) mm two years after surgery

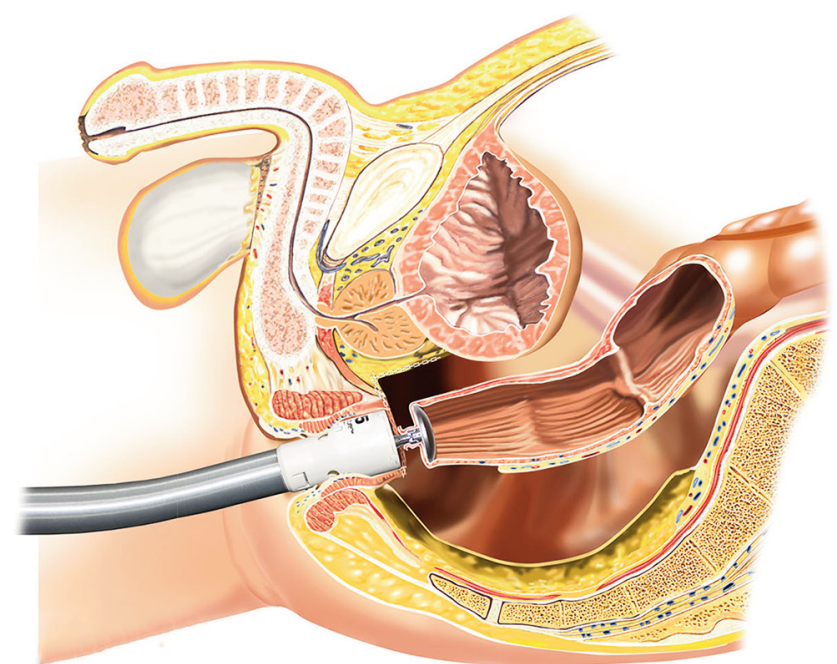

Figure 3. Schema of the J pouch anal anastomosis by a circular stapler. $J$ pouch anal anastomosis is performed by the double stapling technique using a 25-mm circular stapler through the anus.

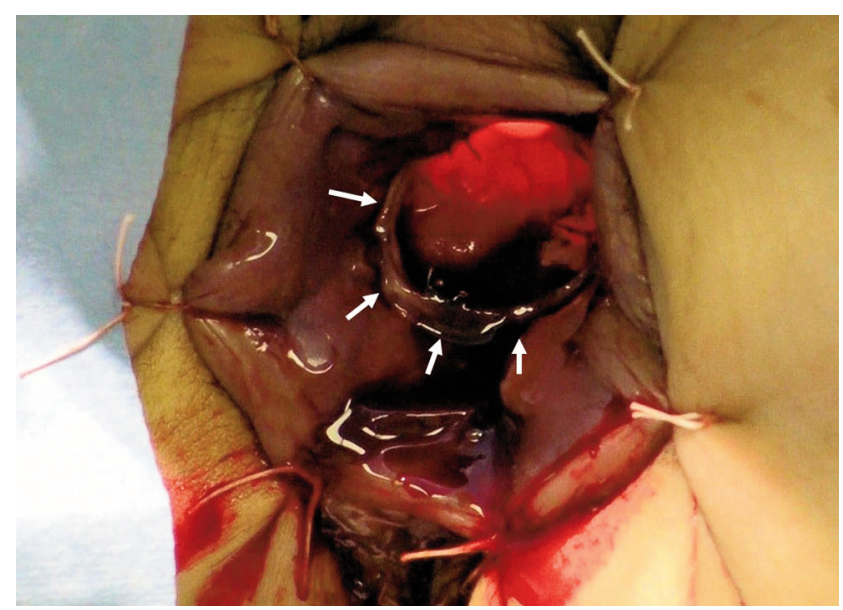

Figure 4. View of J pouch anal anastomosis. An anastomosis which is pointed out with white arrows is created at the dentate line.

Table I. Charactaristics of the patients.

\begin{tabular}{lc}
\hline Variable & $\mathrm{n}=14$ \\
\hline Age (years) & $40.7(19-77)$ \\
Gender & $10(71)$ \\
$\quad$ Male & $4(29)$ \\
Female & $287.2(238-335)$ \\
Duration (min) & $35.0(0-200)$ \\
Bleeding (ml) & $16.4(14-18)$ \\
Postoprerative hospital stay (days) & $3(21)$ \\
Concomitant cancer & \\
\hline
\end{tabular}

The data are presented as mean (range) or as $\mathrm{n}(\%)$. 
Table II. Anorectal function after surgery.

\begin{tabular}{lccc}
\hline Variable & $\begin{array}{c}\text { One year } \\
\text { after surgery }\end{array}$ & $\begin{array}{c}\text { Two years } \\
\text { after surgery }\end{array}$ & $p$-Value \\
\hline $\begin{array}{c}\text { Maximum resting } \\
\text { pressure (mm Hg) }\end{array}$ & $71.7(52-85)$ & $75.3(54-88)$ & 0,427 \\
$\begin{array}{l}\text { Maximum squeeze } \\
\text { pressure (mm Hg) }\end{array}$ & $112.4(87-141)$ & $125.0(90-160)$ & 0.117 \\
$\begin{array}{l}\text { Length of high-pressure } \\
\text { zone (mm) }\end{array}$ & $33.4(30-35)$ & $39.6(35-42)$ & $<0.001$ \\
$\begin{array}{l}\text { Wexner score } \\
\text { Texne }\end{array}$ & $5.0(4-6)$ & $2.8(1-4)$ & $<0.001$ \\
\hline
\end{tabular}

The data are presented as mean (range) or as $\mathrm{n}(\%)$.

$(p<0.001)$. Wexner score was $5.0(4-6)$ one year after surgery and $2.8(1-4)$ two years after surgery $(p<0.001)$ (Table II).

\section{Discussion}

Two types of ileal pouch anal anastomosis have been widely performed for UC patients worldwide. One is a handsewn technique with mucosectomy (4), and the other is a stapling technique with the retention of the mucosa of the rectal stump (5). For the handsewn technique with mucosectomy, all colorectal mucosa is removed to avoid the risk of further inflammatory disease, dysplasia, or cancer. However, the excision of the anal transition zone is associated with postoperative problems of continence (6). On the other hand, for the stapling technique with retention of mucosa, the surgical procedure is simple and provides better functional results compared with the handsewn technique. However, the retention of mucosa has the potential for disease recurrence or malignant degeneration $(7,8)$.

When rectal transection is attempted immediately above the anal canal with a linear stapler, the anterior wall of the rectum just above the anal canal cannot be transected anatomically (1). In our procedure, TSRJASA, after cutting the rectourethral muscle using a $30-\mathrm{mm}$ length stapler, rectal transection was easily performed immediately above the anal canal using a 30$\mathrm{mm}$ length stapler twice. All colorectal mucosa can be removed by triple stapling resection and stapling anastomosis using a 25-mm circular stapler in TSRJASA. Furthermore, our method provided acceptable defecation function. The measurements obtained by manometry, maximum resting pressure, maximum squeeze pressure, and the length of the high-pressure zone yielded similar results compared to reported normal values (9) at two years after surgery. Recovery of the length of the highpressure zone seems to be significantly involved in the noted Wexner score improvements.

\section{Conclusion}

Further long-term follow-up and large-scale studies are still necessary, but TSRJASA is a useful procedure for UC patients. We expect to indicate this procedure for many UC patients.

\section{Conflicts of Interest}

The Authors declare no conflicts of interest.

\section{Authors' Contributions}

Hidejiro Kawahara collected the data, analysed them statistically, put the results into the current context and wrote the article. Nobuo Omura revised it critically for important intellectual content. All Authors read and approved the final version.

\section{References}

1 Choi JS, Potenti F, Wexner SD, Nam YS, Hwang YH, Nogueras JJ, Weiss EG and Pikarsky AJ: Functional outcomes in patients with mucosal ulcerative colitis after ileal pouch-anal anastomosis by the double stapling technique: is there a relation to tissue type? Dis Colon Rectum 43(10): 1398-1404, 2000. PMID: 11052517. DOI: $10.1007 / \mathrm{BF} 02236636$

2 Kawahara H, Akiba T and Yanaga K: Cuff-less J pouch anal stapling anastomosis for ulcerative colitis. Anticancer Res 37(10): 5743-5745, 2017. PMID: 28982895. DOI: 10.21873/anticanres.12013

3 Kawahara H, Hiramoto Y, Takeda M, Matsumoto N, Misawa T and Yanaga $\mathrm{K}$ : Anthropometric assessment after proctocolectomy due to ulcerative colitis. In Vivo 33(1): 239-243, 2019. PMID: 30587630. DOI: 10.21873/invivo.11466

4 Parks AG and Nicholls RJ: Proctocolectomy without ileostomy for ulcerative colitis. Br Med J 2(6130): 85-88, 1978. PMID: 667572. DOI: $10.1136 / \mathrm{bmj} .2 .6130 .85$

5 Heald RJ and Allen DR: Stapled ileo-anal anastomosis: a technique to avoid mucosal proctectomy in the ileal pouch operation. Br J Surg 73(7): 571-572, 1986. PMID: 3730791. DOI: $10.1002 /$ bjs.1800730719

6 Saigusa N, Kurahashi T, Nakamura T, Sugimura H, Baba S, Konno $\mathrm{H}$ and Nakamura S: Functional outcome of stapled ileal pouch-anal canal anastomosis versus handsewn pouch-anal anastomosis. Surg Today 30(7): 575-581, 2000. PMID: 10930221. DOI: 10.1007/s005950070095

7 Silvestri MT, Hurst RD, Rubin MA, Michelassi F and Fichera A: Chronic inflammatory changes in the anal transition zone after stapled ileal pouch-anal anastomosis: is mucosectomy a superior alternative? Surgery 144(4): 533-7; discussion 537-9, 2008. PMID: 18847636. DOI: 10.1016/j.surg.2008.06.003

8 Lovegrove RE, Constantinides VA, Heriot AG, Athanasiou T, Darzi A, Remzi FH, Nicholls RJ, Fazio VW and Tekkis PP: A comparison of hand-sewn versus stapled ileal pouch anal anastomosis (IPAA) following proctocolectomy: a meta-analysis of 4183 patients. Ann Surg 244(1): 18-26, 2006. PMID: 16794385. DOI: 10.1097/01.sla.0000225031.15405.a3

9 Cuong LM, Van Quyet H, Hung TM, Anh NN, Ha TT, Van Du V, Van Loi D, Khai HHH and Kien VD: Normal values for highresolution anorectal manometry in healthy young adults: evidence from Vietnam. BMC Gastroenterol 21(1): 295, 2021. PMID: 34266417. DOI: 10.1186/s12876-021-01865-8

Received January 5, 2022

Revised February 3, 2022

Accepted February 8, 2022 